\title{
Light composite Higgs from an effective action for technicolor
}

\author{
A. Doff* \\ Universidade Tecnológica Federal do Paraná-UTFPR-COMAT, Via do Conhecimento Km 01, 85503-390, Pato Branco-PR, Brazil \\ A. A. Natale ${ }^{+}$ \\ Instituto de Física Teórica, UNESP, Rua Pamplona, 145, 01405-900, São Paulo-SP, Brazil \\ P. S. Rodrigues da Silva \\ Departamento de Física, Universidade Federal da Paraíba, Caixa Postal 5008, 58051-970, João Pessoa-PB, Brazil
}

(Received 13 February 2008; published 29 April 2008)

\begin{abstract}
We compute an effective action for a composite Higgs boson formed by new fermions belonging to a general technicolor non-Abelian gauge theory, using a quite general expression for the fermionic selfenergy that depends on a certain parameter $(\alpha)$, that defines the technicolor theory from the extreme walking behavior up to the one with a standard operator product expansion behavior. We discuss the values of the trilinear and quadrilinear scalar couplings. Our calculation spans all the possible physical possibilities for mass and couplings of the composite system. In the case of extreme walking technicolor theories we verify that it is possible to have a composite Higgs boson with a mass as light as the present experimental limit, contrary to the usual expectation of a heavy mass for the composite Higgs boson. In this case we obtain an upper limit for the Higgs boson mass, $\left(M_{H} \leq \mathcal{O}(700) \mathrm{GeV}\right.$ for $\left.S U(2)_{\mathrm{TC}}\right)$, and the experimental data on the Higgs boson mass constrain $S U(N)_{\mathrm{TC}}$ technicolor gauge groups to be smaller than $S U(10)_{\mathrm{TC}}$.

DOI: 10.1103/PhysRevD.77.075012

PACS numbers: $11.15 . \mathrm{Tk}, 12.60 . \mathrm{Nz}, 12.60 . \mathrm{Rc}$
\end{abstract}

\section{INTRODUCTION}

In the standard model of elementary particles the fermion and gauge boson masses are generated due to the interaction of these particles with elementary Higgs scalar bosons. Despite its success there are some points in the model as, for instance, the enormous range of masses between the lightest and heaviest fermions and other peculiarities that could be better explained at a deeper level. The nature of the Higgs boson is one of the most important problems in particle physics, and there are many questions that may be answered in the near future by the LHC experiments, such as: Is the Higgs boson, if it exists at all, elementary or composite? What are the symmetries behind the Higgs mechanism?

The possibility that the Higgs boson is a composite state instead of an elementary one is more akin to the phenomenon of spontaneous symmetry breaking that originated from the effective Ginzburg-Landau Lagrangian, which can be derived from the microscopic BCS theory of superconductivity describing the electron-hole interaction (or the composite state in our case). This dynamical origin of the spontaneous symmetry breaking has been discussed with the use of many models, the most popular one being the technicolor (TC) model [1]. Unfortunately we do not know the dynamics that form the scalar bound state, which

\footnotetext{
*agomes@utfpr.edu.br

+natale@ift.unesp.br

psilva@fisica.ufpb.br
}

should play the role of the Higgs boson in the standard model symmetry breaking, and no phenomenologically satisfactory model along this line has been derived up to now.

Most of the models for the spontaneous symmetry breaking of the standard model based on the composite Higgs boson system depends on specific assumptions about the theory particle content and consequently on the dynamics responsible for the bound state formation [2], and one of the questions that we address in this work is how can we make predictions about the effective Higgs Lagrangian without assuming specific models or dynamics? In principle, new fermions are bounded by a new interaction stronger than QCD and originate a composite scalar state whose wave function is a solution of the Bethe-Salpeter equation. In non-Abelian gauge theories this wave function (or the Bethe-Salpeter kernel $\Phi_{\mathrm{BS}}(p, q)$ ) is related to the self-energy of the new fermions [3]

$$
\Sigma\left(p^{2}\right)=\left.\Phi_{\mathrm{BS}}(p, q)\right|_{q \rightarrow 0},
$$

and here we shall assume for this self-energy $(\Sigma(p, \alpha))$ a very general expression that interpolates between all possible scalar wave functions (or all possible non-Abelian gauge group dynamics) as we vary a specific parameter $(\alpha)$ present in this function [4]. When this parameter goes to 1 we obtain a fermionic self-energy that behaves as $\Sigma\left(p^{2}\right) \propto$ $\Lambda^{3} / p^{2}$, which is the usual operator product expansion (OPE) behavior for a gauge theory that develops a dynamical mass scale $\Lambda$ [5]. When $\alpha \rightarrow 0$ the self-energy is the one that appears in the extreme walking technicolor theo- 
ries [6]. Using this self-energy ansatz we can study several properties of the composite Higgs boson in a model independent way [7], as we choose the free parameter $(\alpha)$ which defines the theory to be considered.

Observe that Eq. (1) shows that there is formal relation between the fermion self-energy $\left(\Sigma\left(p^{2}\right)\right)$ and the scalar boson wave function. In principle this means that if we know $\Sigma\left(p^{2}\right)$ we know all the properties of the scalar boson. However, we must keep in mind that the calculation of the effective action is not performed with an exact expression for the self-energy (or scalar wave function), but with a simple approximation of this function which obeys the leading order solution of the Schwinger-Dyson equation (SDE) for the fermion propagator. By leading order SDE in the case of non-Abelian gauge theories we understand that the SDE are solved using as input the bare gauge boson propagator and solely the effect of the running coupling in the vertex function. This approximation is usually assumed as reasonable and has already been tested at higher order for walking technicolor theories [8]. On the other hand we also point out that the effective action is a dressed loop expansion, which is able to capture the nonlinearities of the dynamical symmetry breaking under a controllable approximation, as shown by Cornwall and Shellard in Ref. [9], but if we neglect the next order of the loop expansion and consider the leading order and simple approximation for the fermionic self-energy we will surely have an uncertainty in the boson masses and couplings that we quote.

With the general self-energy (or composite state wave function) we can compute an effective action $(\Omega)$ for composite operators [10] of the effective Higgs system, which is a type of calculation already performed for several specific models (see, for instance, Refs. [11,12]). However the effective potential has not been computed up to now with the general self-energy ansatz that we referred to above. Moreover, the effective potential by itself does not give the full information about the composite Higgs system. The effective action contains a kinetic term, which, as demonstrated by Cornwall and Shellard [9], has the form

$$
\Omega_{K}=\frac{1}{2} \int d^{4} x \frac{\left[\partial_{\mu} \phi(x)\right]^{2}}{\kappa},
$$

where $\phi$ is related to the composite wave function and to obtain a conventional kinetic term we define

$$
\Phi(x)=Z^{-1 / 2} \phi(x),
$$

where $\Phi$ plays the role of the physical field and $Z=\kappa$ acts as a renormalization constant. The constant $Z$ is important to set the right scale in our "Ginzburg-Landau" effective Lagrangian; actually, it will be fundamental to the results in order to provide the right values of the composite scalar boson mass and self-coupling constants. This effective Lagrangian will be useful to set limits on the composite Higgs boson system in a quite general way, and it will be given by $\Omega$ which is composed by the kinetic term $\Omega_{K}$ and the effective potential part $\Omega_{V}$. Another point that, as far as we know, has not been extensively discussed in the literature and we discuss here are the different contributions to the effective potential that come from the new fermions that form the scalar composite state, and the ones that come from ordinary fermions. Both contributions are responsible, as we shall see, for determining the value of the composite Higgs boson mass which, as our result indicates, can be as light as a few hundred $\mathrm{GeV}$, corroborating the results of Ref. [13].

This paper is organized as follows: In Sec. II we discuss the effective potential for composite operators and how the kinetic term of the effective action is generated through the use of the general self-energy ansatz. Section III contains the actual calculation of the effective action. In Sec. IV we gather our results and compute the Higgs boson masses, and in Sec. V we draw our conclusions.

\section{EFFECTIVE ACTION AND FERMION SELF- ENERGY}

The effective action for composite operators [10] $(\bar{\Gamma})$, is a function of the Green functions $G_{i}$, and is stationary with respect to variations of $G_{i}$ :

$$
\frac{\delta \bar{\Gamma}}{\delta G_{i}}=0
$$

The effective potential is defined by

$$
V\left(G_{i}\right) \int d^{4} x=-\left.\bar{\Gamma}\left(G_{i}\right)\right|_{\text {translation invariant }}
$$

In terms of the complete fermion $(S)$ and gauge boson $(D)$ propagators, $V\left(G_{i}\right)$ can be written as

$$
\begin{aligned}
V(S, D)= & -i \int \frac{d^{4} p}{(2 \pi)^{4}} \operatorname{Tr}\left(\ln S_{0}^{-1} S-S_{0}^{-1} S+1\right) \\
& +V_{2}(S, D),
\end{aligned}
$$

where $S_{0}$ (and $D_{0}$ ) stands for the bare fermion (gauge boson) propagator.

$V_{2}(S, D)$ is the sum of all two-particle irreducible vacuum diagrams. The only contribution that we shall consider to $V_{2}(S, D)$ is the one depicted in Fig. 1, and the equation

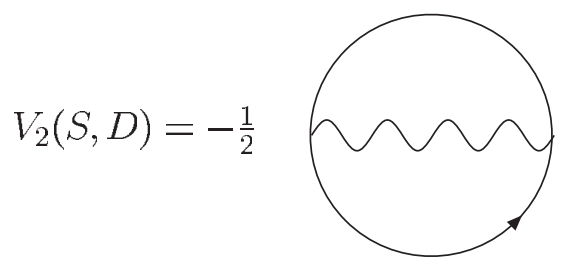

FIG. 1. Two-particle irreducible contribution to the vacuum energy. 


$$
\frac{\delta V}{\delta S}=0,
$$

gives the SDE for the fermion propagator. We are not considering contributions to the potential due to gauge and ghosts loops, because we are interested only in the fermionic bilinear condensation in the scalar channel, keeping in mind that we should consider a non-Abelian gauge theory, stronger than QCD, whose fermions form the composite Higgs boson. Of course, we are also not considering the possibility of gauge boson mass generation in this non-Abelian theory, as may happen in QCD [14], that could imply only in a change of the potential value at the minimum, but not in the symmetry breaking pattern of the effective Higgs theory.

We can represent $V_{2}(S, D)$ analytically in the HartreeFock approximation by

$$
i V_{2}(S, D)=-\frac{1}{2} \operatorname{Tr}(\Gamma S \Gamma S D),
$$

where $\Gamma$ is the fermion proper vertex. In Eq. (8) we have not written the gauge and Lorentz indices, as well as the momentum integrals.

The physically meaningful quantity that we must compute is the vacuum energy density given by

$$
\Omega_{V}=V(S, D)-V\left(S_{0}, D_{0}\right),
$$

where we are subtracting the symmetric part of the potential from the potential that admits condensation in the scalar channel, that is denoted by $V\left(S_{0}, D_{0}\right)$ and is a function of the perturbative propagators $\left(S_{0}\right.$ and $\left.D_{0}\right)$, where the complete propagator $S$ is related to the free propagator by

$$
S^{-1}=S_{0}^{-1}-\Sigma
$$

where $S_{0}=i \not p$.

The vacuum energy density, if we remove all indices and integrations, can be written as $[10,12]$

$$
\begin{aligned}
\Omega_{V}= & -i \operatorname{Tr}\left(\ln S_{0}^{-1} S-S_{0}^{-1} S+1\right)+i \operatorname{Tr} \Sigma\left(S-S_{0}\right) \\
& +\frac{1}{2} i \operatorname{Tr}\left(\Gamma S \Gamma S-\Gamma S_{0} \Gamma S_{0}\right) D .
\end{aligned}
$$

Using Eq. (10) and assuming $\Sigma S_{0}$ small, it is possible to expand $\Omega_{V}$ in powers of $\Sigma$, that gives $[10,12]$

$$
\begin{aligned}
\Omega_{V}= & i \operatorname{Tr} \ln \left(1-\Sigma S_{0}\right)+\frac{1}{2} i \operatorname{Tr} \Sigma S_{0} \Sigma S_{0} \\
& +\frac{1}{2} i \operatorname{Tr} S_{0} \Sigma S_{0} \Sigma S_{0} \Gamma S_{0} \Sigma S_{0} \Sigma S_{0} \Gamma D_{0} .
\end{aligned}
$$

In Eq. (12) we have kept terms only up to the $\Sigma^{4}$ term that comes from the two-loop contribution. Note that expanding the logarithmic term the $\Sigma^{2}$ contribution is absent, which is a consequence of the fact that $\Sigma$ obeys the linear homogeneous SDE for the fermion propagator $[10,15]$.

We parametrize the self-energy $\Sigma$ as [4]

$$
\Sigma\left(p^{2}\right) \sim \Lambda\left(\frac{\Lambda^{2}}{p^{2}}\right)^{\alpha}\left[1+b g^{2} \ln \left(p^{2} / \Lambda^{2}\right)\right]^{-\gamma \cos (\alpha \pi)} .
$$

In the above expression $\Lambda$ is the characteristic scale of mass generation of the theory forming the composite Higgs boson, which hereafter will be identified with the TC scale, $\Lambda_{\mathrm{TC}} . b$ is the coefficient of the $g^{3}$ term in the renormalization group $\beta$ function, $\gamma=3 c / 16 \pi^{2} b$, and $c$ is the quadratic Casimir operator given by

$$
c=\frac{1}{2}\left[C_{2}\left(R_{1}\right)+C_{2}\left(R_{1}\right)-C_{2}\left(R_{3}\right)\right],
$$

where $C_{2}\left(R_{i}\right)$ are the Casimir operators for fermions in the representations $R_{1}$ and $R_{2}$ that form a composite boson in the representation $R_{3}$. The only restriction on this ansatz is $\gamma>1 / 2$ [5], and if we consider the formal equivalence between the solution of the Schwinger-Dyson equation with the Bethe-Salpeter one for scalar bound states, the above restriction indicates a condition on the composite wave-function normalization.

The ansatz in Eq. (13), proposed in Ref. [4], interpolates between the standard OPE result for the technifermion self-energy, which is obtained when $\alpha \rightarrow 1$, and the extreme walking technicolor solution obtained when $\alpha \rightarrow 0$ [6], i.e., this is the case where the symmetry breaking is dominated by higher order interactions that are relevant at or above the TC scale, leading naturally to a very hard dynamics [16,17]. As two of us have pointed out in Ref. [7] only such kind of solution is naturally capable of generating a large mass to the third fermionic generation, which has a mass limit almost saturated by the top quark mass. This variation of the ansatz with $\alpha$ is what makes our calculation a general one; it covers all possible solutions of the Schwinger-Dyson equation (or Bethe-Salpeter equation) for fermions forming the composite boson.

We can now determine a complete effective theory (or the Ginzburg-Landau Lagrangian), including the kinetic term of the effective action. To start with, let us suppose that the real vacuum leads to fermion condensation and denote the true ground state by $|\Omega\rangle$. Taking into account the structure of the real vacuum, the fermion propagators are described by a fermion bilinear which is not translationally invariant

$$
S(x, y)_{\eta \xi}=-i\left\langle\Omega\left|T\left[\chi_{\eta}\left(x+\frac{1}{2} y\right) \psi_{\xi}\left(x-\frac{1}{2} y\right)\right]\right| \Omega\right\rangle .
$$

The Fourier transform of Eq. (14) can be written as

$$
S(p, k)=S_{0}(p, k)+\Sigma(p, k),
$$

where $S_{0}(p, k)$ is the bare propagator (which is translationally invariant) given by

$$
S_{0}(p, k)=(2 \pi)^{4} \delta^{4}(p-k) / k,
$$

and $\Sigma(p, k)$ is a gap equation, which can be separated in its regular part ( $\Sigma_{R}$ - one that does not represent symmetry breaking) and a singular part that breaks the symmetry $\left(\Sigma_{S}(p, k)\right)$

$$
\Sigma(p, k)=(2 \pi)^{4} \Sigma_{R}(k) \delta^{4}(p-k)+\Sigma_{S}(p, k) .
$$


Our ansatz for $\Sigma\left(p^{2}\right)$ that appears in Eq. (13) is nothing else than the linearized solution of $\Sigma(p, k)$.

If we suppose that the expectation value of the fermion bilinear has the following operator expansion [9]

$$
\left\langle\Omega\left|T\left[\chi\left(x+\frac{1}{2} y\right) \psi\left(x-\frac{1}{2} y\right)\right]\right| \Omega\right\rangle \underset{y \rightarrow 0}{\sim} C(y) \phi(x),
$$

where $C(y)$ is a $c$-number function, and $\phi(x)$ acts like a dynamical effective scalar field with anomalous dimension $2 \gamma$. Therefore we can write

$$
\begin{aligned}
\Sigma(p, k) & \sim \phi(k)\left(\frac{\Lambda^{2}}{p^{2}}\right)^{\alpha}\left[1+b g^{2} \ln \left(p^{2} / \Lambda^{2}\right)\right]^{-\gamma \cos (\alpha \pi)} \\
& \equiv \phi(k) \tilde{\Sigma}\left(p^{2}\right) .
\end{aligned}
$$

As seen in Eq. (19), working in the true vacuum generates a nontrivial dependence on the momentum $k$ for our variational parameter $\phi$. The kinetic term for our effective theory is obtained inserting $\phi(k)$ in the effective action and expanding around $k=0$. The diagrams contributing to the kinetic part of the energy density are shown in Fig. 2

\section{THE GINZBURG-LANDAU LAGRANGIAN}

In order to determine the effective Lagrangian we start computing the kinetic term contribution, which is given by the polarization diagrams $\left(\Pi\left(k^{2}, \phi\right)\right)$ of Fig. 2. This contribution is important in our calculation because it will give the correct normalization of the effective fields, as discussed after Eq. (3). The renormalization constant for the scalar composite field is obtained from [9],

$$
\left.Z \approx 2 \frac{d \Pi\left(k^{2}, \phi\right)}{d k^{2}}\right|_{k^{2}=0} .
$$

The Taylor expansion of $Z$ around $k^{2}=0$ gives

$$
\left.Z \approx \frac{k^{2}}{8} g_{\gamma \delta} \frac{\partial}{\partial k_{\gamma}} \frac{\partial}{\partial k_{\delta}} \Pi\left(k^{2}, \phi\right)\right|_{k^{2} \approx 0},
$$

which after some algebra can be written as

$$
\left(Z^{(\alpha)}\right)^{-1} \approx \frac{N_{\mathrm{TC}} n_{F}}{4 \pi^{2}} \int d p^{2} \frac{\left(p^{2}\right)^{2} \tilde{\Sigma}^{2}\left(p^{2}\right)}{\left(p^{2}+\Lambda_{\mathrm{TC}}^{2}\right)^{3}},
$$

where the index $\alpha$ is related to the ansatz of Eq. (19).

Using Eq. (19), considering that the fermions in the loop have technicolor and flavor numbers equal to $N_{\mathrm{TC}}$ and $n_{F}$, respectively, and after some calculation we obtain

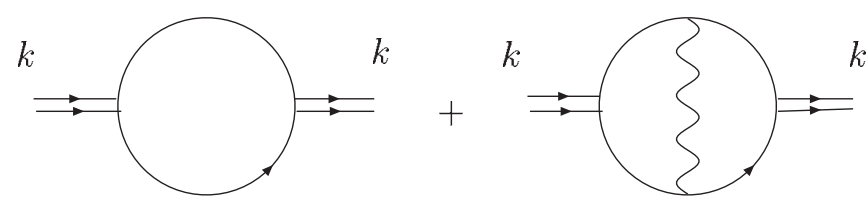

FIG. 2. Diagrams contributing to the kinetic term in the effective Lagrangian.

$$
Z^{(0)} \approx \frac{4 \pi^{2} \beta(2 \gamma-1)}{N_{\mathrm{TC}} n_{F}}\left[1+\frac{\alpha}{\beta(\gamma-1)}+\ldots\right],
$$

where $Z^{(0)}$ is the normalization constant obtained performing the kinetic loop calculation and expanding the result in the limit $\alpha \rightarrow 0$. In Eq. (23) $\beta=b g^{2}$. The limit $\alpha \rightarrow 0$ will correspond to the extreme walking limit of our effective Lagrangian. We do the same calculation for the case $\alpha \rightarrow 1$, obtaining

$$
Z^{(1)} \approx \frac{8 \pi^{2}}{N_{\mathrm{TC}} n_{F}}\left[1-\frac{\beta \gamma}{\alpha}+\ldots\right] .
$$

Our effective Lagrangian will be given by

$$
\Omega^{(\alpha)}=\int d^{4} x\left[\frac{1}{2 Z^{(\alpha)}(\phi)} \partial_{\mu} \phi \partial^{\mu} \phi\right]-\Omega_{V}^{(\alpha)},
$$

where $\Omega_{V}^{(\alpha)}$ can be written in powers of $\phi$ leading to

$$
\begin{aligned}
\Omega^{(\alpha)}= & \int d^{4} x\left[\frac{1}{2 Z^{(\alpha)}(\phi)} \partial_{\mu} \phi \partial^{\mu} \phi-\frac{\lambda_{4 V}^{(\alpha)}}{4} \phi^{4}\right. \\
& \left.-\frac{\lambda_{6 V}^{(\alpha)}}{6} \phi^{6}-\ldots\right],
\end{aligned}
$$

that after renormalization by $Z^{(\alpha)}$ translates to

$$
\Omega_{R}^{(\alpha)}=\int d^{4} x\left[\frac{1}{2} \partial_{\mu} \Phi \partial^{\mu} \Phi-\frac{\lambda_{4 V R}^{(\alpha)}}{4} \Phi^{4}-\frac{\lambda_{6 V R}^{(\alpha)}}{6} \Phi^{6}-\ldots\right] .
$$

In this expression we have defined the renormalized field $\Phi \equiv\left[Z^{(\alpha)}\right]^{-(1 / 2)} \phi$, and the renormalized couplings for the two limits, $\alpha \rightarrow 0$ and $\alpha \rightarrow 1$, are given, respectively, by

$$
\begin{aligned}
\lambda_{4 V R}^{(0)} \equiv & \lambda_{4 V}^{(0)}\left[Z^{(0)}\right]^{2} \\
= & \frac{N_{\mathrm{TC}} n_{F}}{4 \pi^{2}}\left[Z^{(0)}\right]^{2} \times\left[\left(\frac{1}{\beta(4 \delta-1)}+\frac{1}{2}\right)\right. \\
& \left.-\frac{4 \alpha}{\beta(4 \delta-1)}\left(\frac{1}{(4 \delta-2)}+2 \delta\right)\right], \\
\lambda_{6 V R}^{(0)} \equiv & \lambda_{6 V}^{(0)}\left[Z^{(0)}\right]^{3}=-\frac{N_{\mathrm{TC}} n_{F}}{4 \pi^{2}} \frac{\left[Z^{(0)}\right]^{3}}{\Lambda_{\mathrm{TC}}^{2}},
\end{aligned}
$$

and

$$
\begin{aligned}
\lambda_{4 V R}^{(1)} \equiv & \lambda_{4 V}^{(1)}\left[Z^{(1)}\right]^{2} \\
= & \frac{N_{\mathrm{TC}} n_{F}}{4 \pi^{2}}\left[Z^{(1)}\right]^{2} \times\left[\frac{1}{4}\left(1+\frac{c \alpha_{\mathrm{TC}}}{2 \pi}\right)\right. \\
& \left.-\frac{\beta}{4 \alpha}\left(\delta+\frac{c \alpha_{\mathrm{TC}}}{8 \pi}(4 \delta+1)\right)\right], \\
\lambda_{6 V R}^{(1)} \equiv & \lambda_{6 V}^{(1)}\left[Z^{(1)}\right]^{3}=-\frac{N_{\mathrm{TC}} n_{F}}{4 \pi^{2}} \frac{\left[Z^{(1)}\right]^{3}}{7 \Lambda_{\mathrm{TC}}^{2}},
\end{aligned}
$$

where the $\lambda_{n V}^{(\alpha)}$ are the couplings computed in Appendix A.

Note also that besides the absence of a $\phi^{2}$ term, due to the fact that we assumed that our ansatz satisfies the linear 
fermionic self-energy equation, we do not have odd powers of the effective $\phi$ field in the potential because we are assuming massless technifermions [18].

Up to now we have discussed the contributions to the effective Lagrangian that are originated from the new fermions responsible for the composite scalar state. In models based on the technicolor idea the composite scalar boson is made of these new fermions only. Of course there are models like topcolor [19] where the top quark has a strong interaction such as it could supply the scalar composite necessary to the dynamical symmetry breaking of the electroweak theory. This last possibility would change the contributions that we should consider to the potential, but as there is no observed signal in the top quark physics up to now indicating such a possibility, we do not follow this path and consider that our composite state is formed only by new fermionic degrees of freedom. However, even in this case we still have other contributions to the effective Lagrangian. The contributions that we are referring to are the ones coming from ordinary massive quarks and leptons that couple to the scalar boson. These contributions will be dominated by the heaviest fermion (the top quark) and will generate terms of order $\phi^{3}, \phi^{4}$, and higher as will be discussed in the sequence.

The $\phi^{3}$ and $\phi^{4}$ contributions to the effective Lagrangian due to the ordinary massive fermions are given, respectively, by the diagrams of Figs. 3 and 4, where the effective $f f \phi$ coupling is determined through Ward identities as discussed in Refs. $[16,17,20]$, and it is easy to verify that such a coupling will be given by

$$
{ }_{l} \lambda_{\phi f f} \propto-\imath \frac{g_{W} \Sigma_{f}(k)}{2 M_{W}} .
$$

Notice that the ordinary fermions masses in composite Higgs models come from a new type of interaction, that in the most common approach is called extended technicolor interaction (ETC). As we do not know the dynamics (or model) for this specific interaction, we cannot formally derive their contribution to the effective action. However, we can compute the effect of ordinary fermions to the effective potential as a function of their masses, exactly as performed by Carpenter et al. [21]. These contributions are expected to be small, since the ordinary fermion masses

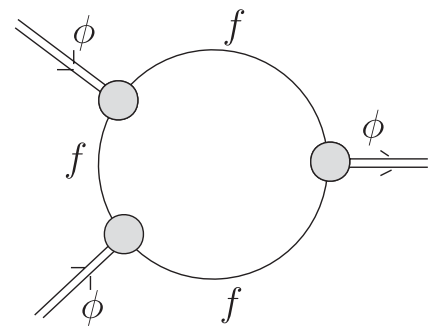

FIG. 3. Heavy ordinary fermions $(f)$ contribution to the trilinear composite $(\phi)$ Higgs boson coupling. The gray blobs are proportional to the effective $f f \phi$ coupling.

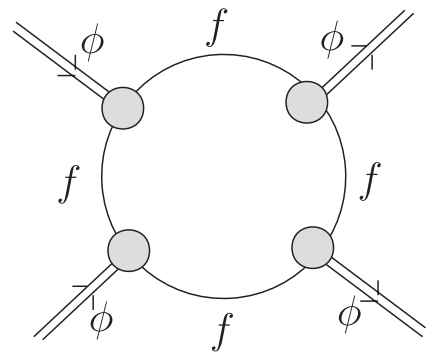

FIG. 4. Heavy ordinary fermions $(f)$ contribution to the quadrilinear composite $(\phi)$ Higgs boson coupling. The vertices are proportional to the effective $f f \phi$ coupling.

are smaller than the characteristic composite scale $\left(\Lambda_{\mathrm{TC}}\right)$. The calculation of the $\phi^{3}$ and $\phi^{4}$ terms are presented in Appendix B, where we determine the effective trilinear and quadrilinear couplings (the contributions to $\Omega_{V}$ are obtained multiplying these couplings by the normalized fields). The couplings are equal to:

(a) Trilinear coupling when $\alpha \sim 0$

$$
\begin{aligned}
\lambda_{3 f}^{(0)} \approx & \frac{9 g_{W}^{3}}{32 \pi^{2}} \frac{m_{t}}{\beta(4 \delta-1)}\left(\frac{m_{t}}{M_{W}}\right)^{3} \\
& \times\left[1-\frac{4 \alpha}{\beta(4 \delta-2)}+\ldots\right],
\end{aligned}
$$

(b) Trilinear coupling when $\alpha \sim 1$

$$
\lambda_{3 f}^{(1)} \approx \frac{9 g_{W}^{3}}{32 \pi^{2}} \frac{m_{u}}{4}\left(\frac{m_{u}}{M_{W}}\right)^{3}\left[1-\frac{\beta(4 \delta-1)}{4 \alpha}+\ldots\right]
$$

(c) Quadrilinear coupling when $\alpha=0$

$$
\lambda_{4 f}^{(0)} \approx \frac{3 g_{W}^{4}}{64 \pi^{2} M_{W}^{4}} \frac{m_{t}^{4}}{\beta(4 \delta-1)},
$$

This result is the same as the one obtained by Carpenter et al. [21].

(d) Quadrilinear coupling when $\alpha=1$

$$
\lambda_{4 f}^{(1)} \approx \frac{3 g_{W}^{4}}{64 \pi^{2} M_{W}^{4}} \frac{m_{u}^{4}}{4} .
$$

The fact that when $\alpha \sim 1$ we introduced the mass $m_{u}$, as discussed in Appendix B, is an approximation, because in this case we can only generate light fermion masses, in order to be consistent with the absence of flavor changing neutral currents. Actually we should say that this last case is not important and should not be considered, since a relevant contribution would come from heavy fermions, and as far as it is known up to now [7], such heavy mass could only be naturally generated in extreme walking gauge theories. 
Observe that the above Eqs. (31)-(34) can easily be rewritten in terms of the TC scale $\Lambda_{\mathrm{TC}}$ if we use the relation between $M_{W}$ and the technipion constant $F_{\Pi}$,

$$
M_{W}^{2}=\frac{g_{w}^{2} n_{d} F_{\Pi}^{2}}{4},
$$

where $n_{d}$ is the number of technifermion doublets and $F_{\Pi}$ is obtained from the Pagels and Stokar relation [22],

$$
\begin{aligned}
F_{\Pi}^{2}= & \frac{N_{T C}}{8 \pi^{2}} \int \frac{d p^{2} p^{2}}{\left(p^{2}+\Sigma^{2}\left(p^{2}\right)\right)^{2}}\left[\Sigma^{2}\left(p^{2}\right)\right. \\
& \left.-\frac{p^{2}}{2} \frac{d \Sigma\left(p^{2}\right)}{d p^{2}} \Sigma\left(p^{2}\right)\right] .
\end{aligned}
$$

After transforming the above momentum integral in Eq. (36) through a Mellin transformation,

$$
\left[1+\beta \ln \frac{p^{2}}{\Lambda_{\mathrm{TC}}^{2}}\right]^{-2 \delta}=\frac{1}{\Gamma(2 \delta)} \int_{0}^{\infty} d z z^{2 \delta-1} e^{-z}\left(\frac{p^{2}}{\Lambda_{\mathrm{TC}}^{2}}\right)^{-\beta z},
$$

and using Eq. (13) and the following expression for the factor $Z$

$$
\left(Z^{(\alpha)}\right)^{-1} \approx \frac{N_{\mathrm{TC}} n_{F}}{4 \pi^{2}} \frac{1}{\Gamma(2 \delta)} \int_{0}^{\infty} d z \frac{z^{2 \delta-1} e^{-z}}{(2 \alpha+\beta z)},
$$

we can rewrite the equation for $F_{\Pi}$ in terms of $Z^{(\alpha)}$, which leads to

$$
n_{d} F_{\Pi}^{2}=\left(1+\frac{\alpha}{2}\right) \frac{\Lambda_{\mathrm{TC}}^{2}}{Z^{(\alpha)}} .
$$

Equation (39) is an interesting example of how the technipion decay constant varies with the theory dynamics (or with $\alpha$ ). Notice that if we change the dynamics of the theory we cannot obtain $F_{\Pi}^{2}$ just with a simple scaled QCD. Another interesting fact is also the relation between $F_{\Pi}^{2}$ and $Z^{(\alpha)}$. Since the fields in the effective Lagrangian are normalized by different powers of $Z^{(\alpha)}$ (or powers of $F_{\Pi}^{2}$, that also varies with $\alpha$ ), and since $F_{\Pi}^{2}$ is fixed by the weak gauge boson masses, we verified that the behavior of the effective theory is quite different according to the different limits of the $\alpha$ parameter.

\section{RESULTS}

The full effective Lagrangian for the composite Higgs system will be given by

$$
\Omega=\int d^{4} x\left[\frac{1}{2 Z^{(\alpha)}} \partial_{\mu} \phi \partial^{\mu} \phi\right]-\Omega_{V} .
$$

Introducing the normalized field

$$
\Phi=\phi\left[Z^{(\alpha)}\right]^{-1 / 2},
$$

we can write

$$
\begin{aligned}
\Omega_{R}^{(\alpha)}= & \int d^{4} x\left[\frac{1}{2} \partial_{\mu} \Phi \partial^{\mu} \Phi-\frac{\lambda_{3 f R}^{(\alpha)}}{3} \Phi^{3}\right. \\
& \left.-\frac{\left(\lambda_{4 V R}^{(\alpha)}+\lambda_{4 f R}^{(\alpha)}\right)}{4} \Phi^{4}-\frac{\lambda_{6 V R}^{(\alpha)}}{6} \Phi^{6}+\ldots\right] .
\end{aligned}
$$

The coupling constants that appear in Eq. (42) are the ones obtained in the previous section. It must be noticed that the couplings originated from the ordinary fermion masses are smaller than the ones generated from the techniquarks effective potential. For example,

$$
\frac{\lambda_{4 V}^{(0)}}{\lambda_{4 f}^{(0)}} \approx \frac{\left(N_{\mathrm{TC}} n_{F}\right)^{3}}{12\left(16 \pi^{2} \beta(2 \gamma-1)\right)^{2}}\left(\frac{\Lambda_{\mathrm{TC}}}{m_{t}}\right)^{4} .
$$

The $\beta(2 \gamma-1)$ factor appearing in the denominator is usually of $\mathcal{O}(1)$ for several gauge groups. Assuming a $S U(4)_{\text {TC }}$ technicolor theory with $n_{F}=14$ [23], in the case when $\alpha \rightarrow 0$, we will obtain a ratio of the following order

$$
\frac{\lambda_{4 V}^{(0)}}{\lambda_{4 f}^{(0)}} \approx \mathcal{O}(10) .
$$

In the case when $\alpha \rightarrow 1$ the difference can be even larger. This means that we can neglect the ordinary massive fermions contribution to $\Omega_{R}^{(\alpha)}$ (proportional to $\lambda_{n f}^{(\alpha)}$ ) compared to the one of techniquarks (proportional to $\lambda_{n V}^{(\alpha)}$ ). The only exception is the $\left(\lambda_{3 f R}^{(\alpha)} / 3\right) \Phi^{3}$ term, which is small but is the leading term of this order in the effective action and introduces some effect in the scalar mass calculation.

We can now compute the scalar mass which is determined from the following equation:

$$
M_{\Phi}^{2(\alpha)}=\left.\frac{\partial^{2} \Omega_{R}^{(\alpha)}}{\partial \Phi^{2}}\right|_{\Phi=\Phi_{\min }} .
$$

After neglecting terms proportional to $\lambda_{6}$ and of higher order when substituting the minimum value in the potential we obtain

$$
M_{\Phi}^{2(\alpha)} \approx 2 \lambda_{4 V R}^{(\alpha)}\left(\frac{\lambda_{4 V R}^{(\alpha)}}{\lambda_{6 V R}^{(\alpha)}}\right)+5 \lambda_{3 f R}^{(\alpha)}\left(\frac{\lambda_{4 V R}^{(\alpha)}}{\lambda_{6 V R}^{(\alpha)}}\right)^{1 / 2} .
$$

With Eq. (46) we can compute numerically the Higgs boson mass in the extreme walking behavior $(\alpha \rightarrow 0)$ and the result is plotted in Fig. 5. Notice that as we go to larger values of $N_{\mathrm{TC}}$ while keeping a slowly TC running coupling constant (a $\beta_{\text {TC }}$ function close to zero) we verify that the current experimental limit on the Higgs boson mass does not allow us to have a technicolor gauge group arbitrarily large $\left(N_{\mathrm{TC}}<10\right)$. The possibility that a composite Higgs boson can be as light as the present experimental limit has been already noticed in a series of papers [13]. The authors of these papers particularly discuss a more interesting case where the walking behavior is obtained in theories where 


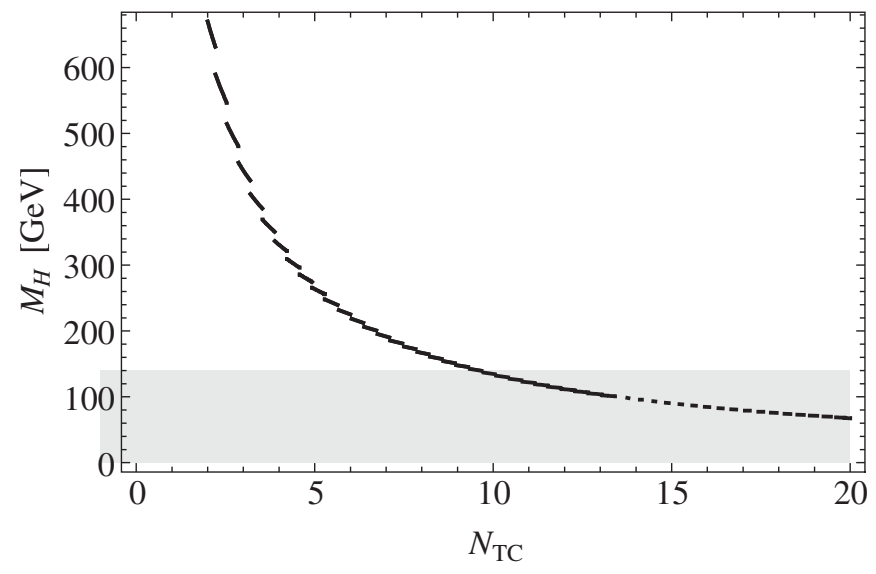

FIG. 5. Higgs mass as a function of $N_{\mathrm{TC}}$ in the extreme walking technicolor regime. The shaded band is the experimentally excluded region [25].

the fermions are in higher dimensional representations of the technicolor group, turning unnecessary the introduction of a quite large number of fermions, as happens in the case where the fermions are in the fundamental representation. Moreover, it was also shown that exactly for the extreme walking case these theories, with a light composite Higgs, are totally in agreement with the precision electroweak measurements [13]. In obtaining Fig. 5 we have used the $\beta$ function up to two loops, where $n_{F}$ for each $S U\left(N_{\mathrm{TC}}\right)$ has to be fixed accordingly, i.e., $n_{F}=8,11,14, \ldots$ for $N_{\mathrm{TC}}=$ $2,3,4, \ldots$

Considering the smallest possible non-Abelian unitary technicolor gauge group, i.e., $S U(2)_{\mathrm{TC}}$, we can observe from Fig. 5 that, in the extreme walking regime, the Higgs boson mass has an upper limit of about $\mathcal{O}(700) \mathrm{GeV}$. In order to have models with dynamical symmetry breaking along the technicolor idea without the problems of neutral flavor changing currents, the walking scenario seems to be the most promising possibility [6]. In this context our result implies a crucial test for the walking technicolor hypothesis since such mass values may be promptly assessed at LHC.

Let us consider the limit $\lambda_{3 f R}^{(\alpha)} \rightarrow 0$. In this case we obtain the conventional result for $M_{\Phi}^{2(\alpha)}$ given by the effective potential

$$
M_{\Phi}^{2(\alpha)} \approx 2 \frac{\left[\lambda_{4 V}^{(\alpha)}\right]^{2}}{\lambda_{6 V}^{(\alpha)}} .
$$

We can observe that the top quark mass $\left(m_{t} \sim 175 \mathrm{GeV}\right)$ will usually give a contribution of the order of $10 \%$ of the composite Higgs boson mass through the trilinear Higgs boson coupling. We show in Fig. 6 some values for the Higgs mass versus the trilinear coupling for some technicolor models already discussed in the literature [6]. The points that we have chosen in Fig. 6 correspond to extreme walking technicolor theories, and we expect the possible range of couplings and masses for other $\alpha$ values to be

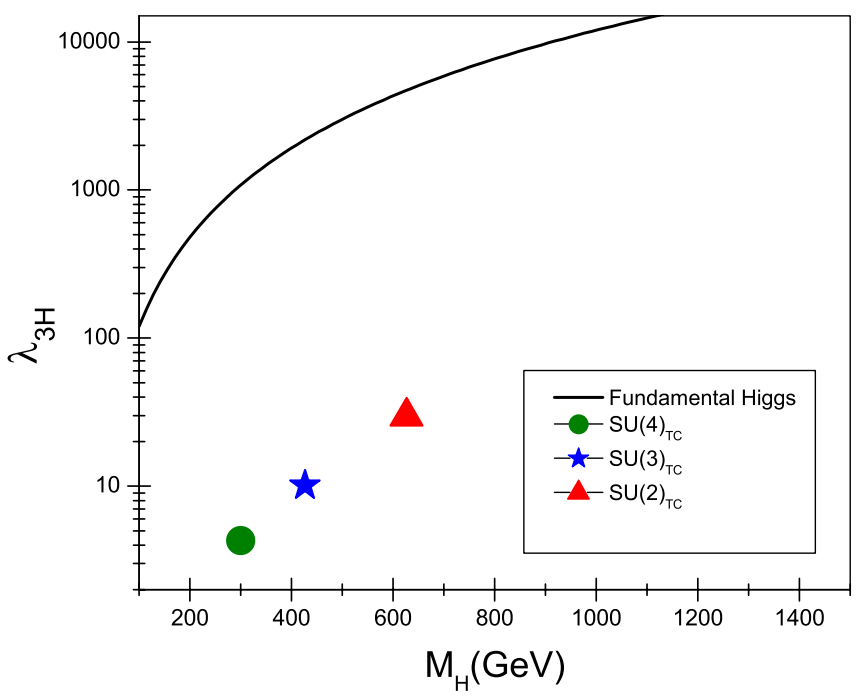

FIG. 6 (color online). Trilinear scalar coupling as a function of composite boson mass. We plot a solid line with the standard model value for this relation and show the expected values for a composite Higgs boson based on $S U(2)_{\mathrm{TC}}, S U(3)_{\mathrm{TC}}$, and $S U(4)_{\mathrm{TC}}$ models, with $n_{f}=8,11,14$, respectively.

located between these points and the standard model curve [7].

Finally, in the limit $\alpha \rightarrow 1$ we simply obtain scalar boson masses in the $\mathrm{TeV}$ region as usual, but these models are known to be plagued by unwanted flavor changing neutral currents.

\section{CONCLUSIONS}

We have computed an effective action for a composite Higgs boson system formed by new fermions belonging to a general technicolor non-Abelian gauge theory. The calculation is based on an effective action for composite operators. The novelty is that the effective action is computed with the help of a quite general self-energy that depends on a certain parameter $(\alpha)$, which, when variated from 0 to 1, provides an interpolation of the fermionic selfenergy from the extreme walking technicolor behavior up to the self-energy expression that obeys the standard operator product expansion. This means that our calculation is quite general in the sense that choosing values for $\alpha$, which is equivalent to choosing different dynamics for the strong interaction forming the composite scalar boson, we can obtain the different mass and couplings of the effective theory.

There are two other improvements in our calculation. The first one is the calculation of the kinetic term of the effective theory. This term appears with a coefficient that differs from the standard parametrization of the kinetic term of a scalar Lagrangian. When the effective Lagrangian is normalized to reproduce a standard scalar effective field theory we also must modify the remaining terms, leading to a nontrivial change of the scalar self- 
couplings. The second improvement is that we also consider the effect of ordinary massive fermions to the effective Lagrangian. This contribution is usually neglected and is indeed small except by the contribution of the heavy top quark. An ordinary massive fermion contribution is also important because it introduces odd powers of scalar field self-couplings in the theory, i.e., the trilinear composite scalar self-coupling is originated from the loop of the top quark, while the quadrilinear self-coupling is dominated by the techniquarks interactions as well as, in a minor extent, from the top quark loop contribution. Of course, this result would change if there is a fourth ordinary fermion family or if the techniquarks have a large current mass (above the TC scale).

With the general fermionic self-energy (or composite state wave function) we computed the effective Lagrangian presenting the results for $\alpha=0$ and $\alpha=1$, which correspond to the limits of the extreme walking technicolor theory and the standard view of the technicolor theory that can be obtained by scaled QCD. For other $\alpha$ values the scalar mass and self-couplings are located between the ones obtained for the extreme cases $(0$ and 1$)$. In the case of an extreme walking behavior $(\alpha \rightarrow 0)$, we obtain an upper limit for the Higgs boson mass, $\left(M_{H} \leq \mathcal{O}(700) \mathrm{GeV}\right.$ for $\left.S U(2)_{\mathrm{TC}}\right)$, and the experimental data on the Higgs boson mass constrain $S U(N)_{\text {TC }}$ technicolor gauge groups to be smaller than $S U(10)_{\mathrm{TC}}$, whereas when $\alpha \rightarrow 1$ the scalar mass is expected to be much heavier. Therefore we agree with the earlier results of Ref. [13] that we may have quite a light composite Higgs scalar boson in the case of extreme walking TC theories.

It is fair to mention that another source of uncertainty in our approach, besides that assumed in the SDE, is that we are showing results for the extreme walking behavior $(\alpha \rightarrow$ 0 ), for which we consider the $\beta$ function up to 2 loops. This obviously constrains the number of fermions introduced in our computation of the effective potential. Higher loops certainly change the number of fermions needed to get the walking behavior, implying a change in our numerical results for the triple and quartic gauge couplings as well as the Higgs mass. It is possible that going to further orders of the beta function could modify the specific shape of the Higgs mass function shown in Fig. 5 and shift the couplings relative to the expected results of the standard model plotted in Fig. 6. Given the degree of approximations we have already assumed in computing the effective potential, we thought it was reasonable to truncate the beta function to the order that its coefficients are universal. However, it is interesting to notice that a complete all orders beta function obtained in Ref. [24] could be used in a more general approach and also when different representations are considered and could be helpful in developing an extended analysis in a future work.

In theories where the scalar Higgs boson is composite we need new "extended technicolor" interactions in order to give masses to the ordinary fermions. As far as we know there is no phenomenologically viable ETC model and its effect enters in our effective Lagrangian parametrized in the massive ordinary fermion contributions. This contribution is important, as discussed above, because it is responsible for the trilinear scalar coupling and we expect that other ETC contributions decouple from the effective Lagrangian. The ordinary fermion contributions to the effective Lagrangian are roughly 1 order of magnitude smaller than the one of techniquarks. This is an expected behavior since their masses are smaller than the TC mass scale.

At present the walking technicolor models seem to be the most promising possibility for dynamically broken gauge theories. Therefore if this scenario is appropriate to describe the dynamics of symmetry breaking (with a unitary gauge group in the fundamental representation), our limit implies that the scalar composite boson should be observed at LHC with a mass up to $700 \mathrm{GeV}$, a quite interesting outcome considering a composite nature for the Higgs boson.

\section{ACKNOWLEDGMENTS}

This research was partially supported by the Conselho Nacional de Desenvolvimento Científico e Tecnológico $(\mathrm{CNPq})$.

\section{APPENDIX A: $\phi^{4}$ AND $\phi^{6}$ CONTRIBUTIONS TO $\mathbf{\Omega}_{V}$}

In this appendix we compute the $\phi^{4}$ and $\phi^{6}$ terms of the effective potential $\Omega_{V}$. We start from the effective action up to two loops (see Eq. (12)):

$$
\begin{aligned}
\Omega_{V}^{(\alpha)}= & i \operatorname{Tr} \ln \left(1-\Sigma S_{0}\right)+\frac{1}{2} i \operatorname{Tr} \Sigma S_{0} \Sigma S_{0} \\
& +\frac{1}{2} i \operatorname{Tr} S_{0} \Sigma S_{0} \Sigma S_{0} \Gamma S_{0} \Sigma S_{0} \Sigma S_{0} \Gamma D_{0},
\end{aligned}
$$

where the last term comes from the two-loop contribution.

Expanding the term proportional to $\ln \left(1-\Sigma S_{0}\right)$, considering the propagators, vertices, and the $\Sigma$ ansatz with the momentum dependence of Eq. (19), we obtain

$$
\begin{aligned}
\Omega_{V}^{(\alpha)}= & \frac{N_{\mathrm{TC}} n_{F}}{16 \pi^{2}} \frac{1}{\Gamma(4 \delta)} \int \frac{d z z^{4 \delta-1} e^{-z}}{(4 \alpha+\beta z)} \operatorname{Tr}\left(\phi^{4}\right) \\
& +\frac{N_{\mathrm{TC}} n_{F}}{16 \pi^{2}} \frac{3 \alpha_{\mathrm{TC}} c}{4 \pi(2+4 \alpha)} \frac{4}{\Gamma(4 \delta+1)} \\
& \times \int \frac{d z z^{(4 \delta+1)-1} e^{-z}}{(4 \alpha+\beta z)} \operatorname{Tr}\left(\phi^{4}\right) \\
& +\frac{N_{\mathrm{TC}} n_{F}}{16 \pi^{2}} \operatorname{Tr}\left[\phi^{4}\left(\sum_{m=1} \frac{\left(\frac{\phi^{2}}{\Lambda_{\mathrm{TC}}^{2}}\right)^{m}}{(2 m+4)} \frac{(-1)^{m}}{(m+(2 m+4) \alpha)}\right)\right],
\end{aligned}
$$

where the contribution of $\mathcal{O}\left(\Sigma^{2}\right)$ is canceled between the two first terms of Eq. (A1), and the last term is what remains of the $\ln \left(1-\Sigma S_{0}\right)$ expansion after cancellation 
of the $\Sigma^{2}$ contribution and extraction of the $\Sigma^{4}$ contribution.

We can compute Eq. (A2) in the limits $\alpha=0$ and $\alpha=$ 1 . In the case $\alpha \approx 0$ we have

$$
\begin{aligned}
\Omega_{V}^{(0)}= & \frac{N_{\mathrm{TC}} n_{F}}{16 \pi^{2}}\left[\left(\frac{1}{\beta(4 \delta-1)}+\frac{1}{2}\right)\right. \\
& \left.-\frac{4 \alpha}{\beta(4 \delta-1)}\left(\frac{1}{(4 \delta-2)}+2 \delta\right)\right] \operatorname{Tr}\left(\phi^{4}\right) \\
& +\frac{N_{\mathrm{TC}} n_{F}}{16 \pi^{2}}\left[-\Lambda_{\mathrm{TC}}^{4} \operatorname{Tr}\left(\frac{\phi^{2}}{\Lambda_{\mathrm{TC}}^{2}}\right)+\frac{\Lambda_{\mathrm{TC}}^{4}}{2} \operatorname{Tr}\left(\frac{\phi^{4}}{\Lambda_{\mathrm{TC}}^{4}}\right)\right] \\
& +\frac{N_{\mathrm{TC}} n_{F}}{16 \pi^{2}}\left[\operatorname{Tr}\left[\left(1-\frac{\phi^{4}}{\Lambda_{\mathrm{TC}}^{4}}\right) \ln \left(1+\frac{\phi^{2}}{\Lambda_{\mathrm{TC}}^{2}}\right)\right]\right] .
\end{aligned}
$$

If we assume $\frac{\phi^{2}}{\Lambda_{\mathrm{TC}}^{2}} \ll 1$ we obtain

$$
\begin{aligned}
\Omega_{V}^{(0)}= & \frac{N_{\mathrm{TC}} n_{F}}{16 \pi^{2}}\left[\left(\frac{1}{\beta(4 \delta-1)}+\frac{1}{2}\right)\right. \\
& \left.-\frac{4 \alpha}{\beta(4 \delta-1)}\left(\frac{1}{(4 \delta-2)}+2 \delta\right)\right] \operatorname{Tr}\left(\phi^{4}\right) \\
& -\frac{N_{\mathrm{TC}} n_{F}}{16 \pi^{2}}\left[\frac{2}{3 \Lambda_{\mathrm{TC}}^{2}}\right] \operatorname{Tr}\left(\phi^{6}\right)+O\left(\operatorname{Tr}\left(\phi^{8}\right)\right) \ldots
\end{aligned}
$$

In the limit $\alpha \approx 1$ we have

$$
\begin{aligned}
\Omega_{V}^{(1)}= & \frac{N_{\mathrm{TC}} n_{F}}{16 \pi^{2}}\left[\frac{1}{4}\left(1+\frac{c \alpha_{\mathrm{TC}}}{2 \pi}\right)\right. \\
& \left.-\frac{\beta}{4 \alpha}\left(\delta+\frac{c \alpha_{\mathrm{TC}}}{8 \pi}(4 \delta+1)\right)\right] \operatorname{Tr}\left(\phi^{4}\right)+\frac{N_{\mathrm{TC}} n_{F}}{16 \pi^{2}} \\
& \times\left[\Lambda_{\mathrm{TC}}^{4} \operatorname{Tr}\left(\frac{\phi^{2}}{\Lambda_{\mathrm{TC}}^{2}}\left[2-3_{2} F_{1}\left(1, \frac{1}{3} ; \frac{4}{3} ;-\frac{\phi^{2}}{\Lambda_{\mathrm{TC}}^{2}}\right)\right]\right)\right] \\
& +\frac{N_{\mathrm{TC}} n_{F}}{16 \pi^{2}}\left[-\frac{\Lambda_{\mathrm{TC}}^{4}}{4} \operatorname{Tr}\left(\frac{\phi^{4}}{\Lambda_{\mathrm{TC}}^{4}}\right)+\Lambda_{\mathrm{TC}}^{4} \ln \left(1+\frac{\phi^{2}}{\Lambda_{\mathrm{TC}}^{2}}\right)\right],
\end{aligned}
$$

where $p F_{q}\left(a_{1}, \ldots, a_{p} ; b_{1}, \ldots b_{q} ; x\right)$ is the hypergeometric function.

Again assuming $\frac{\phi^{2}}{\Lambda_{\mathrm{TC}}^{2}} \ll 1$ we obtain the following $\phi^{4}$ and $\phi^{6}$ contributions to $\Omega_{V}^{(1)}$

$$
\begin{aligned}
\Omega_{V}^{(1)}= & \frac{N_{\mathrm{TC}} n_{F}}{16 \pi^{2}}\left[\frac{1}{4}\left(1+\frac{c \alpha_{\mathrm{TC}}}{2 \pi}\right)\right. \\
& \left.-\frac{\beta}{4 \alpha}\left(\delta+\frac{c \alpha_{\mathrm{TC}}}{8 \pi}(4 \delta+1)\right)\right] \operatorname{Tr}\left(\phi^{4}\right) \\
& -\frac{N_{\mathrm{TC}} n_{F}}{16 \pi^{2}}\left[\frac{2}{21 \Lambda_{\mathrm{TC}}^{2}}\right] \operatorname{Tr}\left(\phi^{6}\right)+O\left(\operatorname{Tr}\left(\phi^{8}\right)\right) \ldots
\end{aligned}
$$

From Eqs. (A4) and (A6) we can read the values of the couplings $\lambda_{4 V}^{(0)}$ and $\lambda_{4 V}^{(1)}$, which are given, respectively, by

$$
\lambda_{4 V}^{(0)} \approx \frac{N_{\mathrm{TC}} n_{F}}{16 \pi^{2}}\left(\frac{1}{\beta(4 \delta-1)}+\frac{1}{2}\right),
$$

$$
\lambda_{4 V}^{(1)} \approx \frac{N_{\mathrm{TC}} n_{F}}{16 \pi^{2}} \frac{1}{4}\left(1+\frac{c \alpha_{\mathrm{TC}}}{2 \pi}\right) .
$$

The $(1 / 2)$ factor at the end of Eq. (A7) comes from the two-loop contribution.

In the same way, as done above for the $\lambda_{4 V}^{(\alpha)}$ coupling, we can easily obtain the $\lambda_{6 V}^{(\alpha)}$ from Eqs. (A4) and (A6). These results are the ones shown in the Sec. III.

\section{APPENDIX B: TRILINEAR AND QUADRILINEAR COUPLINGS ORIGINATED FROM ORDINARY FERMIONS}

The trilinear and quadrilinear couplings that are originated from the ordinary massive fermions are obtained from the calculation of Figs. 3 and 4, respectively. Assuming that the coupling $\phi \bar{f} f$, of the composite Higgs scalar boson $\phi$ to the ordinary fermions, at large momentum $p^{2}$ is given by [17]

we obtain

$$
\lambda_{\phi f f} \approx-\frac{g_{W} \Sigma_{f}\left(p^{2}\right)}{2 M_{W}}
$$

$$
\lambda_{4 f}^{(\alpha)} \approx \frac{1}{64 \pi^{2}} \frac{g_{W}^{4} n_{F} N_{c}}{\left(M_{W}\right)^{4}} \int \frac{d p^{2} p^{6} \Sigma_{f}^{4}\left(p^{2}\right)}{\left(p^{2}+m_{f}^{2}\right)^{4}},
$$

where in this expression $\Sigma_{f}\left(p^{2}\right)$ is parametrized by the ansatz of Eq. (13). Moreover, the infrared cutoff $\Lambda$, which is the characteristic scale of the mass generation in Eq. (13), in this case will be identified with $\Lambda=m_{f}$ exactly as performed in Ref. [17]. After some calculation we can write, in the limit $\alpha=0$, the following quadrilinear coupling

$$
\lambda_{4 f}^{(0)} \approx \frac{3 g_{W}^{4}}{64 \pi^{2} M_{W}^{4}} \frac{m_{f}^{4}}{\beta(4 \delta-1)} .
$$

The largest contribution comes from the heaviest fermion, which can be identified with the top quark $\left(m_{f}=m_{f}(0) \approx\right.$ $\left.m_{t}\right)$ or the lepton tau $\left(m_{f}=m_{f}(0) \approx m_{\tau}\right)$, if we consider leptons. We do the same calculation for the case $\alpha=1$, obtaining

$$
\lambda_{4 f}^{(1)} \approx \frac{3 g_{W}^{4}}{64 \pi^{2} M_{W}^{4}} \frac{m_{f}^{4}}{4} .
$$

The self-energy solution, in this specific limit, cannot generate large fermion masses [7] (without generating large flavor changing neutral currents). Therefore we can expect that $\left(m_{f}=m_{f}(1) \approx m_{u}\right)$ or $\left(m_{f}=m_{f}(1) \approx m_{e}\right)$. The trilinear self-coupling of the composite Higgs bosons with the ordinary fermions can be obtained in the same way, and the result is

$$
\lambda_{3 f}^{(\alpha)} \approx \frac{3 g_{W}^{3} n_{F} N_{c}}{\left(M_{W}\right)^{3}} \frac{1}{32 \pi^{2}} \int \frac{d p^{2} p^{4} \Sigma_{f}^{4}\left(p^{2}\right)}{\left(p^{2}+m_{f}^{2}\right)^{3}}
$$

where for $\alpha \sim 0$ and $\alpha \sim 1$ we obtain

$$
\lambda_{3 f}^{(0)} \approx \frac{9 g_{W}^{3}}{32 \pi^{2}} \frac{m_{f}(0)}{\beta(4 \delta-1)}\left(\frac{m_{f}(0)}{M_{W}}\right)^{3}\left[1-\frac{4 \alpha}{\beta(4 \delta-2)}+\ldots\right]
$$




$$
\lambda_{3 f}^{(1)} \approx \frac{9 g_{W}^{3}}{32 \pi^{2}} \frac{m_{f}(1)}{4}\left(\frac{m_{f}(1)}{M_{W}}\right)^{3}\left[1-\frac{\beta(4 \delta-1)}{4 \alpha}+\ldots\right] .
$$

The couplings shown in Eqs. (B2)-(B6) are the ones appearing in Eqs. (31)-(34).
[1] K. Lane, Technicolor 2000, Lectures at the LNF Spring School in Nuclear, Subnuclear and Astroparticle Physics (Frascati, Rome, Italy, 2000); arXiv:hep-ph/0007304; see also arXiv:hep-ph/0202255;R.S. Chivukula, Models of Electroweak Symmetry Breaking, NATO Advanced Study Institute on Quantum Field Theory Perspective and Prospective (Les Houches, France, 1998); Dynamical Gauge Symmetry Breaking, edited by E. Farhi and R. Jackiw (World Scientific, Singapore, 1982).

[2] C. T. Hill and E. H. Simmons, Phys. Rep. 381, 235 (2003); 390, 553(E) (2004).

[3] R. Delbourgo and M. D. Scadron, Phys. Rev. Lett. 48, 379 (1982).

[4] A. Doff and A. A. Natale, Phys. Lett. B 537, 275 (2002).

[5] K. Lane, Phys. Rev. D 10, 2605 (1974).

[6] B. Holdom, Phys. Rev. D 24, 1441 (1981); Phys. Lett. B 150, 301 (1985); T. Appelquist, D. Karabali, and L. C. R. Wijewardhana, Phys. Rev. Lett. 57, 957 (1986); T. Appelquist and L. C. R. Wijewardhana, Phys. Rev. D 36, 568 (1987); K. Yamawaki, M. Bando, and K. I. Matumoto, Phys. Rev. Lett. 56, 1335 (1986); T. Akiba and T. Yanagida, Phys. Lett. B 169, 432 (1986).

[7] A. Doff and A. A. Natale, Phys. Rev. D 68, 077702 (2003).

[8] T. Appelquist, K. D. Lane, and U. Mahanta, Phys. Rev. Lett. 61, 1553 (1988); U. Mahanta, Phys. Rev. Lett. 62, 2349 (1989); A. G. Cohen and H. Georgi, Nucl. Phys. B314, 7 (1989).

[9] J. M. Cornwall and R. C. Shellard, Phys. Rev. D 18, 1216 (1978).

[10] J. M. Cornwall, R. Jackiw, and E. Tomboulis, Phys. Rev. D 10, 2428 (1974).

[11] M.E. Peskin, in Recent Advances in Field Theory and Statistical Mechanics, edited by J. B. Zuber and R. Stora (Elsevier, New York, 1984).

[12] A. A. Natale, Nucl. Phys. B226, 365 (1983).

[13] F. Sannino, Int. J. Mod. Phys. A 20, 6133 (2005); D. D. Dietrich, F. Sannino, and K. Tuominen, Phys. Rev. D 72, 055001 (2005); N. Evans and F. Sannino, arXiv:hep-ph/ 0512080; D. D. Dietrich, F. Sannino, and K. Tuominen, Phys. Rev. D 73, 037701 (2006); D. D. Dietrich and F. Sannino, Phys. Rev. D 75, 085018 (2007); R. Foadi, M. T.
Frandsen, T. A. Ryttov, and F. Sannino, Phys. Rev. D 76, 055005 (2007); R. Foadi, M. T. Frandsen, and F. Sannino, arXiv:hep-ph/0712.1948 [Phys. Rev. D (to be published)].

[14] J. M. Cornwall, Phys. Rev. D 26, 1453 (1982); A. C. Aguilar and A. A. Natale, J. High Energy Phys. 08 (2004) 057; A. C. Aguilar and J. Papavassiliou, J. High Energy Phys. 12 (2006) 012.

[15] J. M. Cornwall and R. E. Norton, Phys. Rev. D 8, 3338 (1973).

[16] J. Carpenter, R. Norton, S. Siegemund-Broka, and A. Soni, Phys. Rev. Lett. 65, 153 (1990).

[17] J. D. Carpenter, R. E. Norton, and A. Soni, Phys. Lett. B 212, 63 (1988).

[18] Odd powers of the effective potential only appear when massive fermions are introduced. This was studied in detail, in the QCD case, by A. Barducci et al., Phys. Rev. D 38, 238 (1988).

[19] V. A. Miransky, M. Tanabashi, and K. Yamawaki, Phys. Lett. B 221, 177 (1989); V. A. Miransky, M. Tanabashi, and K. Yamawaki, Mod. Phys. Lett. A 4, 1043 (1989); William A. Bardeen, Christopher T. Hill, and Manfred Lindner, Phys. Rev. D 41, 1647 (1990); Christopher T. Hill, Phys. Lett. B 266, 419 (1991); William A. Bardeen and Christopher T. Hill, Adv. Ser. Dir. High Energy Phys. 10, 649 (1992); Christopher T. Hill, Phys. Lett. B 345, 483 (1995); Bogdan A. Dobrescu and Christopher T. Hill, Phys. Rev. Lett. 81, 2634 (1998); R. S. Chivukula, Bogdan A. Dobrescu, Howard Georgi, and Christopher T. Hill, Phys. Rev. D 59, 075003 (1999).

[20] A. Doff and A. A. Natale, Phys. Lett. B 641, 198 (2006).

[21] Notice that their result was obtained in the context of an Abelian gauge theory dominated by high order interactions at high energy (see Ref. [16]).

[22] H. Pagels and S. Stokar, Phys. Rev. D 20, 2947 (1979).

[23] Here we are considering a two-loop $\beta$ function close to an ultraviolet fixed point, which does not jeopardize our previous considerations when proposing the ansatz equation (13).

[24] T. A. Ryttov and F. Sannino, arXiv:hep-th/0711.3745.

[25] T. Aaltonen et al. (CDF Collaboration), arXiv:hep-ex/ 0802.0432 . 